\title{
The Simple Underlying Dynamics of Globular Clusters
}

\author{
Ivan R. King \\ Department of Astronomy, University of Washington, \\ Box 351580, Seattle, WA 98195-1580, USA \\ email: king@astro.washington.edu
}

\begin{abstract}
Although the overall dynamics of globular clusters involves many complexities, much of their dynamics can be understood on the basis of some simple and straightforward physical arguments. Those arguments are presented here, in an effort to lead the reader through the basic stages of cluster dynamics in a quick and easy way.
\end{abstract}

Keywords. globular clusters: general, open clusters and associations: general

\section{Introduction}

The dynamics of globular clusters has many complexities: binaries, collapsed cores, tidal tails, etc., but underlying it all is a basic dynamical structure that is very simple. My aim in this paper is to lead the reader through this underlying structure in a straightforward and logical way.

This is therefore a simple paper, with a simple thesis, but one that I think is very important to an astronomer who wishes to understand the dynamics of globular clusters. The picture that I will draw here includes the essence of cluster dynamics - the inevitable submission to the physical laws and processes that make a cluster what it is. The physical arguments presented here are simple ones, such as any first-year graduate student should be able to follow easily. Yet I fear that in this era of facile computation some graduate students are never exposed to the simple initiation in intuitive physics that I am going to present.

What I will do is to use all of the clarity of hindsight to show how the fundamental characteristics of globular clusters, that is, their velocity distributions and density distributions, are the inevitable outcome of some simple physical processes that govern their behavior, and that it is these processes that make the clusters what they are.

More specifically, I will show how the characteristic family of density profiles of globular clusters follows inevitably and naturally from the relaxing effect of stellar encounters. First, the velocity distribution and the spatial density distribution must match in a way that keeps the cluster in dynamical equilibrium. Then we will see how relaxation through stellar encounters brings the velocity distribution as close to Gaussian as it can get, and how the result is the type of density distributions that clusters have, differing only in central concentration.

The approach that I take here will draw heavily on material from two papers of many decades ago (King 1965 and King 1966). The second of these presented some simple dynamical models of clusters; they turned out to fit the density profiles of globular clusters surprisingly well. (I say surprisingly, because they were the first thing that I tried.) But here, instead of just saying "This works", I will present the models as the inevitable unfolding of the underlying physics. In spite of all the complexity that cluster dynamics appears to have, I want to show that whatever may happen with the details, 
clusters had to follow the models that are derived in the King (1966) paper, and that this becomes obvious if we look at the physics in the right way.

Finally, to end the introduction, some simplifications: I will confine my discussion to the dynamics of a spherical cluster in which all the motions are isotropic. Dealing with a flattened, rotating cluster is not necessary to a basic understanding; and I will dispense with anisotropy too, on the grounds that where it has been measured it has been found to be quite small (King \& Anderson 2001, 2002), so that it is unlikely to be of fundamental importance.

\section{Dynamical Equilibrium}

A self-gravitating star cluster must be in equilibrium. This requires not just satisfying the virial theorem; the velocity distribution has to correspond to the spatial density distribution in just such a way that as the individual stars move around on their orbits, stars take the place of other stars in a way that maintains the density distribution in a constant state.

There is a straightforward algorithm for going from the velocity distribution at the center of a cluster to a complete picture of the velocities and densities throughout the cluster - that is, to a cluster model. Jeans' theorem says that the phase-space density of a system must be expressible as a function of the isolating integrals of the equations of motion of a star. A consequence of this is that from an assumed velocity distribution at the cluster center, we can get the isotropic velocity distribution that holds at every other point. Knowing the velocities, we can then integrate over them to get the density as a function of potential, which comes in through our use of Jeans' theorem. If we put that information into Poisson's equation, we get the relationship between the potential $V$ and the radial coordinate $r$, and closing the circle between $\rho, V$, and $r$ gives us $\rho(r)$, which is the other half of the model. So this algorithm for building a cluster model from a velocity distribution is quite straightforward.

To be more explicit, here are the details. In general, the motion of a star in a spherical potential has two isolating integrals, the energy and the magnitude of the angular momentum. But if we restrict ourselves to isotropic velocity distributions, the only integral that remains is the energy,

$$
E=\frac{1}{2} v^{2}+V(r)
$$

According to Jeans' theorem, then, the joint distribution of density and velocity, $f(r, v)$, is given everywhere by $f(r, v)=F(E)$, where $F$ is some fixed function. This allows us to integrate the density at any point from the formal relationship

$$
\begin{aligned}
\rho & =4 \pi \int_{0}^{v_{\mathrm{e}}} f(r, v) v^{2} d v \\
& =\frac{4 \pi}{\sqrt{2}} \int_{V}^{0} F(E) \frac{d E}{\sqrt{E-V}} .
\end{aligned}
$$

(Note: The limits of integration have been adjusted to recognize the existence of an escape velocity.)

Equation (2.1) allows us to evaluate the right side of Poisson's equation,

$$
\frac{d^{2} V}{d r^{2}}+\frac{2}{r} \frac{d V}{d r}=4 \pi G \rho,
$$

for any value of $V$. The equation can then be solved numerically to give the relationship 
between $V$ and $r$. Along with the relationship between $\rho$ and $V$ given by Eq. (2.1), this gives us $\rho(r)$, which is the explicit spatial density distribution that corresponds to the velocity distribution that we started with. Thus a velocity distribution implies a unique and explicit density distribution. The two distributions together constitute what we call a cluster model.

\section{The Choice of Velocity Distribution}

We have just shown that any reasonable velocity distribution will lead to a valid cluster model. But the question is, which velocity distribution to choose. The obvious answer would seem to be the one toward which the physics of stellar encounters makes the stars of the cluster tend: the Gaussian distribution. But that turns out to go too far. If we put a Gaussian velocity distribution into the algorithm that I have just described, the resulting spatial density distribution is the isothermal sphere, whose mass is strongly infinite - strongly, in the sense that far from the center, the total mass within a radius $r$ increases linearly with $r$.

It is easy to see that something like this had to be so, because a Gaussian distribution has no velocity limit and thus implies an infinite escape velocity. Or, looking at it the other way round, a cluster can never achieve a Gaussian distribution, because its stars of highest velocity would then be above the escape velocity, and would therefore escape.

(An interesting sidelight is that nearly 70 years ago Ambartsumian [1938, 1985] estimated the escape rate by making a very simple approximation. He said to imagine that a cluster achieves a full Gaussian distribution each relaxation time, and that the stars that are above the escape velocity then escape. This crude calculation gave the first estimate of the rate at which stars escape from a cluster, and it was a good one.)

Getting back to our cluster-model problem, what we need is a velocity distribution that is lower than Gaussian at the higher velocities. One could imagine fiddling a Gaussian arbitrarily to reduce its tail, but there is a more natural approach to the problem.

\subsection{The steady-state distribution}

What our cluster is most likely to do is to settle down into a steady state - that is, its velocity distribution will try to take on a steady state, or rather it will get as close as it can get to a steady state, given the fact that stars are slowly escaping. The spatial density distribution will then have whatever form corresponds to that.

The steady state follows from the equation that governs the changes that stellar encounters make in the velocity distribution, the Fokker-Planck equation. Let the stars encountered have a Gaussian velocity distribution,

$$
f_{\mathrm{b}}(v)=\frac{j_{\mathrm{b}}^{3}}{\pi^{3 / 2}} e^{-j_{\mathrm{b}}^{2} v^{2}},
$$

and express the time in units of the "reference time", given by

$$
t_{\mathrm{R}}=\frac{1}{2 \pi G^{2} m_{\mathrm{b}}^{2} n_{\mathrm{b}} j_{\mathrm{b}}^{2} \ln (N / 2)} .
$$

(It is interesting to note that whereas earlier treatments of the theory of stellar encounters had used a quantity called the relaxation time, with an arbitrary definition such as, for example, "the time in which the expected cumulative energy change for an average star is equal to the average kinetic energy of a star", in the case of the Fokker-Planck equation the quantity $t_{\mathrm{R}}$ arises naturally, simply as a collection of all the constants in such a way that defining $\theta=t / t_{\mathrm{R}}$ as a dimensionless time will remove all those constants 
from the equation. The value of $t_{\mathrm{R}}$ is almost the same as that of the relaxation time, within a constant of the order of unity, but to emphasize the fact again, it falls naturally out of the physics.)

In these simplified variables the Fokker-Planck equation takes the form

$$
\frac{\partial f}{\partial \theta}=\frac{1}{x^{2}} \frac{\partial}{\partial x}\left[\alpha(x)\left(\frac{\partial f}{\partial x}+2 \frac{m}{m_{\mathrm{b}}} x f\right)\right],
$$

where $x=j_{\mathrm{b}} v$ and $\alpha(x)$ is defined by

$$
\alpha(x)=\frac{4}{\sqrt{\pi} x} \int_{0}^{x} e^{-y^{2}} y^{2} d y .
$$

To find the steady-state solution we write

$$
f(x, \theta)=g(x) \cdot h(\theta) .
$$

The separation equation is

$$
\frac{1}{h} \frac{d h}{d \theta}=-\lambda=\frac{1}{g} \cdot \frac{1}{x^{2}} \frac{d}{d x}\left[\alpha\left(\frac{d g}{d x}+2 \frac{m}{m_{\mathrm{b}}} x g\right)\right] .
$$

The eigenvalue $\lambda$ (whose minus sign was chosen with foreknowledge), must be a constant, since one equal sign shows it to be independent of $x$ and the other shows it to be independent of $\theta$.

The time-dependence solution is clearly $h=h_{0} e^{-\lambda \theta}$. Thus the solution that corresponds to the smallest eigenvalue will decay most slowly and will at late times become dominant. $\dagger$

The eigenvalue is found by solving the equation

$$
\frac{d}{d x}\left[\alpha\left(\frac{d g}{d x}+2 \frac{m}{m_{\mathrm{b}}} x g\right)\right]+\lambda x^{2} g=0 .
$$

Spitzer and Härm (1958), who were the first to write the Fokker-Planck equation in this form, solved it numerically. It is more instructive, though, to derive an analytic approximation to the steady-state solution of the Fokker-Planck equation, because that leads to a useful approximate formula for the velocity distribution itself (King 1965). It goes as follows:

The equation for $g$ can be integrated formally to give

$$
\frac{d g}{d x}+2 \frac{m}{m_{\mathrm{b}}} x g=-\frac{\lambda}{\alpha(x)} \int_{0}^{x} g(y) y^{2} d y .
$$

If we expand $g(x)$ as a power series in the small quantity $\lambda$,

$$
g(x)=g_{0}(x)+\lambda g_{1}(x)+\lambda^{2} g_{2}(x)+\ldots,
$$

and if we substitute in the differential equation and then equate the coefficients of like

$\dagger$ A note for the students (and perhaps for professors too): In the courses that I took in graduate school, it was standard practice just to do a separation of variables - no explanation; that's just "what one does". So I will interject the explanation here: It can be shown that the equation below is a member of the class called Sturm-Liouville equations. These have the property that their eigenfunctions are a complete basis set, so that any initial velocity distribution can be expressed as a linear combination of them. In that expansion the coefficients are the corresponding $h$ functions. Thus as time goes on, the term with the slowest time decay becomes dominant. This is the justification for saying that the steady-state solution is really the solution that the cluster will choose. 
powers of $\lambda$, the results are

$$
\begin{aligned}
\frac{d g_{0}}{d x}+2 \frac{m}{m_{\mathrm{b}}} x g_{0} & =0 \\
\frac{d g_{i+1}}{d x}+2 \frac{m}{m_{\mathrm{b}}} x g_{i+1} & =-\frac{1}{\alpha(x)} \int_{0}^{x} g_{i}(y) y^{2} d y, \quad i>0 .
\end{aligned}
$$

If we do the same with the boundary conditions, then $g(0)=1$ gives $g_{0}(0)=1$, and $g_{i}(0)=0$ if $i>0$, while the requirement that $(d g / d x)_{x=0}=0$ gives $\left(d g_{i} / d x\right)_{x=0}=0$ for all $i$.

An integrating factor for our equations is $\exp \left(m x^{2} / m_{\mathrm{b}}\right)$. The results are rather complicated, except for the case $m=m_{\mathrm{b}}$, which gives an elegant and simple result. In that case the first equation gives

$$
g_{0}=e^{-x^{2}} .
$$

In the equation for $g_{1}$, the indefinite integral is the same as the one in the definition of $\alpha$, so that the equation simplifies as follows. (To avoid all that annoying filling in of steps in one's head, I have laid it all out, step by step.)

$$
\begin{aligned}
\frac{d g_{1}}{d x}+2 x g_{1} & =-\frac{\sqrt{\pi}}{4} x \\
e^{x^{2}}\left(\frac{d g_{1}}{d x}+2 x g_{1}\right) & =-\frac{\sqrt{\pi}}{4} x e^{x^{2}} \\
\frac{d}{d x}\left(e^{x^{2}} g_{1}\right) & =-\frac{\sqrt{\pi}}{4} x e^{x^{2}} \\
g_{1} & =-\frac{\sqrt{\pi}}{4} e^{-x^{2}} \int_{0}^{x} x e^{x^{2}} d x \\
& =-\frac{\sqrt{\pi}}{8} e^{-x^{2}}\left(e^{x^{2}}-1\right) \\
& =-\frac{\sqrt{\pi}}{8}\left(1-e^{-x^{2}}\right) .
\end{aligned}
$$

We will thus take as our approximate solution

$$
\begin{aligned}
g(x) & =g_{0}(x)+\lambda g_{1}(x) \\
& =e^{-x^{2}}-\frac{\sqrt{\pi}}{8} \lambda\left(1-e^{-x^{2}}\right) .
\end{aligned}
$$

The value of $\lambda$ is set by the condition $g\left(x_{\mathrm{e}}\right)=0$. This gives

and

$$
\lambda=\frac{8}{\sqrt{\pi}} \frac{e^{-x_{\mathrm{e}}^{2}}}{1-e^{-x_{\mathrm{e}}^{2}}}
$$

$$
g(x)=\frac{e^{-x^{2}}-e^{-x_{\mathrm{e}}^{2}}}{1-e^{-x_{\mathrm{e}}^{2}}} .
$$

For $x_{\mathrm{e}}^{2}=6$, the value for which Spitzer and Härm did their numerical integration, this approximation for $\lambda$ comes out $1 / 89.4$; their numerical solution gave 1/88.0. And our approximate $g(x)$ is everywhere within $\frac{1}{2} \%$ of their numerical solution.

The distribution function has a simple and convenient form: a Gaussian lowered by a constant that brings it to zero at $x=x_{\mathrm{e}}$, and renormalized so that $g(0)=1$. 


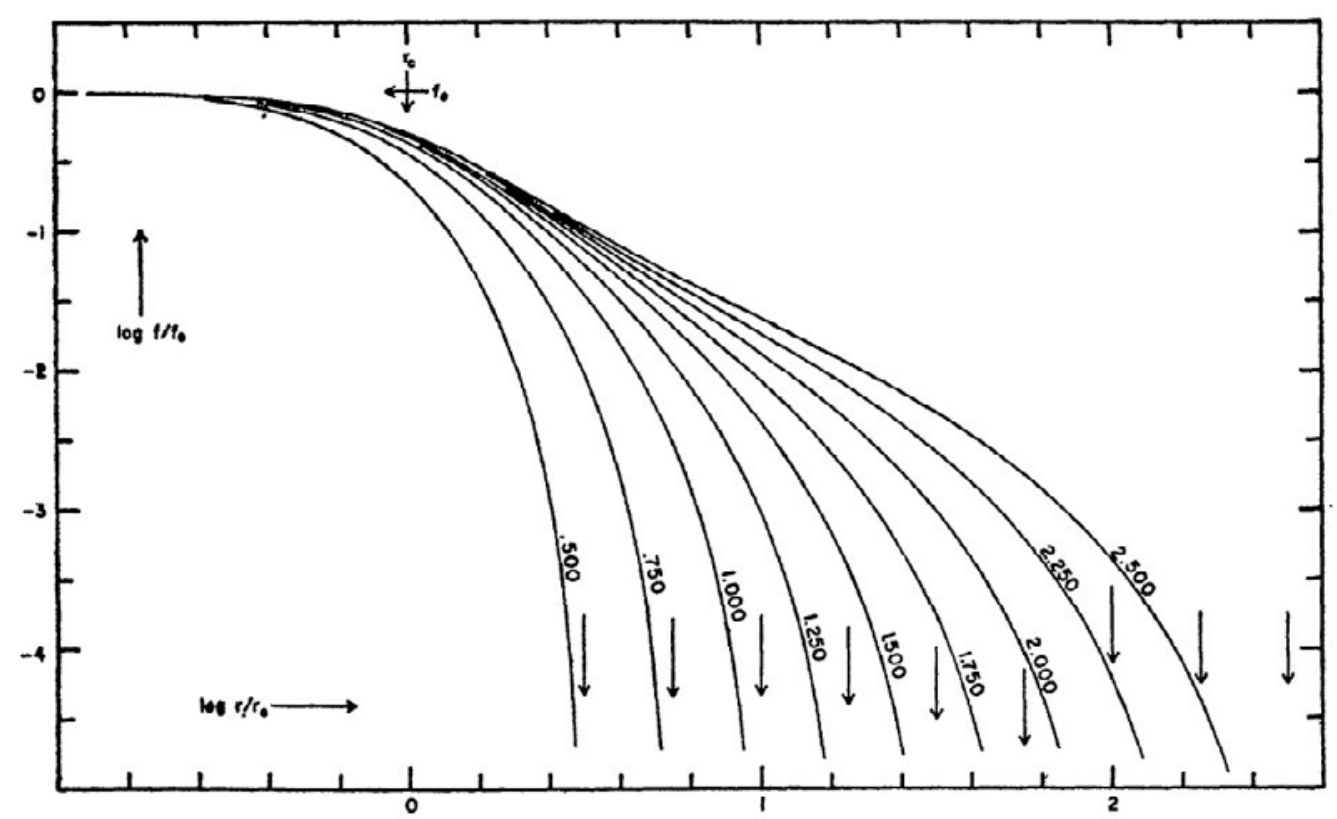

Figure 1. Surface densities in the family of models discussed in the text. All the curves are normalized to the same central surface brightness and core radius. The vertical arrows indicate the limiting radii of successive models.

Notice that $x_{\mathrm{e}}$ has not been specified. In fact, we can evaluate escape-rate eigenvalues and velocity-distribution eigenfunctions for any value of $x_{\mathrm{e}}$, and we get a one-parameter family of solutions, as shown in Fig. 1.

\section{Escape Velocity and the Tidal Limit}

It is now time to attach a physical meaning to another quantity. All of our velocity distributions have been limited so as to go to zero at an "escape velocity". Similarly, the spatial distributions that follow from solving Poisson's equation have a limiting radius. But we can of course attach a physical meaning to this radius in a real cluster; it is just the tidal limit of the cluster in the gravitational field of the Milky Way. (Strictly speaking, the tidal limit is a rigorously defined quantity only for a cluster in a circular orbit, but the idea can be stretched a little to cover non-circular orbits.)

Now consider the problem of making a globular cluster. First you take your celestial ice-cream scoop and decide how much mass to use. Then you decide how closely to cram it together; this gives the core radius of the cluster. Then let the Milky Way impose its tidal force; that sets the tidal limit. These conditions define the actual cluster. The cluster model, on its side, is described by three parameters: $r_{\mathrm{c}}, r_{\mathrm{t}}$, and the mass. Since two of these are just scale factors, the only free parameter is the ratio of $r_{\mathrm{t}}$ to $r_{\mathrm{c}}$, whose logarithm is often called the central concentration. In the picture that we have just drawn, clusters differ from each other only in scale factors and in this one profile parameter, so that they are as similar to each other as they can possibly be.

These models do fit the profiles of actual globular clusters reasonably well, as shown in Fig. 2. 

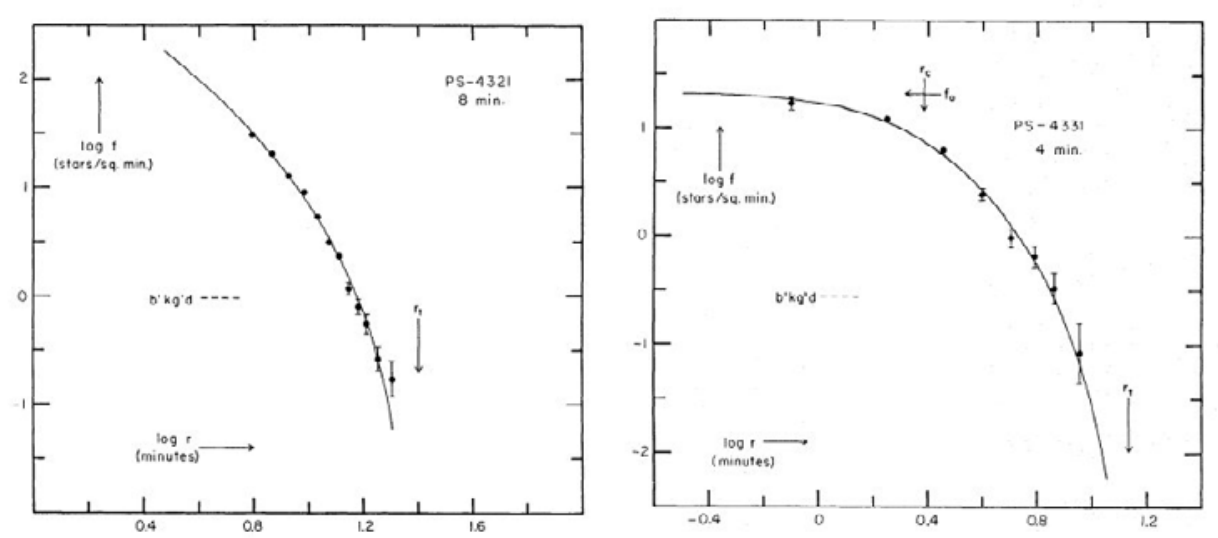

Figure 2. Fitting of model curves to star counts in actual globular clusters. Error bars shown are statistical uncertainties in the counts, from the Poisson distribution. (left) M13; near the center the stars were too crowded to count. (right) NGC 5053.

\section{Additional Favorable Characteristics}

In addition to their inevitability, these models have an unusual stability, in two ways, neither of which had to be so a priori. You may have noticed that it was only at the cluster center that we imposed the requirement that the velocity distribution be the steady-state solution of the Fokker-Planck equation. In a configuration that is going to have long-term stability, however, the condition should apply everywhere. But lo and behold! If we use Jeans' theorem to find out what the velocity distribution is at some other radial distance, it turns out to be the steady-state solution of the Fokker-Planck equation that is appropriate for that point. So the steady state holds everywhere, and the rate of change of the cluster is less than it would be if this were not the case.

The second unexpected equilibrium is in the escape rate of stars. If a different fraction escaped at each distance from the center, the cluster would need to need to readjust, so as to reestablish its dynamical equilibrium. In fact, however, over a large part of the cluster the outward decrease of the escape rate (due to the lower density) is almost exactly balanced by the increased fraction of stars escaping per relaxation time, which is due to the lower escape velocity at radii closer to the tidal limit.

These two circumstances make the dynamical equilibrium much more stable than it would otherwise be, but I have never seen any obvious reason why either of these favorable characteristics should have come about.

\section{A Summing Up}

This is the basic picture that I wanted to present. Globular clusters look so much like each other because they are all molded by the same simple physics. Dynamical systems like to settle down into as steady a state as they can. First, the velocity distribution and the spatial density distribution must be "matched", in the sense that each sustains the other and they remain in an equilibrium. Second, stellar encounters drive the velocity distribution as close to Gaussian as it can get. The form that the velocity distribution takes on is the steady-state solution of the Fokker-Planck equation, and it is that distribution that gives the cluster the characteristic density profile that it has. Third, the remaining physical constraint is imposed by the tidal force of the Milky Way, and within these restrictions the clusters are as similar as they can be. 


\section{Back to Reality}

But I owe you more realism than this. The dynamical models that I have been talking about are oversimplified in one terribly important way: they consist of stars that all have the same mass, whereas a cluster is a mixture of objects of different mass. How do we cope with this? Well, it turns out not to be a serious complication at all. Gravitational forces are additive, so all that we need to do to make a model for a mixture of masses is to write Poisson' equation as

$$
\frac{d^{2} V}{d r^{2}}+\frac{2}{r} \frac{d V}{d r}=4 \pi \sum_{i} G \rho_{i},
$$

where the $\rho_{i}$ refer to the different mass groups. Given the velocity distribution for each mass group, we can evaluate its density at each value of $V$, add up the densities, and do the same integration of Poisson's equation as before.

The only new problem that has come in is getting the velocity distributions for the stars of each mass. I have shown that for stars that all have the same mass the velocity distribution is well approximated by a Gaussian minus a constant. For other masses the solution is not quite so easy, but the velocity distribution is still pretty well approximated by a Gaussian minus a constant. And for the various Gaussians we just take dispersions that correspond to equipartition between the various masses. Because the gravitational potential is pretty well dominated by stars that don't have a very large range in mass, the spatial distribution comes out looking not very different from that of stars of a single mass, and the picture doesn't change very much. And in going through this line of reasoning we have built ourselves a model of how the different stellar masses are distributed relative to each other, so that someone who observes a mass function in one part of a cluster now knows how to go from that local mass function to the global mass function for the whole cluster. A lot more can be learned from this simple picture, like how fast stars of different mass escape, or how tidal shocks affect stars of different mass.

It was stated above that a model made from a mixture of masses usually has a brightness profile that is similar to that of some member of the single-mass family. There is one striking exception, however: a model with a very small core. Such models arise when the central concentration is high and there is a significant number of stars that have a significantly higher mass than any of the other groups - for example, if about $1 \%$ the stars in a globular-cluster model have masses suitable for neutron stars $\left(\sim 1.4 M_{\odot}\right)$. In that case the greater masses of the neutron stars cause them to bunch up in the center and create a lump-like concentration of mass there. In such a model the number of neutron stars can be adjusted to make the brightness profile of the model closely resemble that of a collapsed-core cluster. This ability of the models is valuable to note when, for instance, transforming a local mass function to a global mass function in a collapsed-core cluster.

Now I will say a little about one other aspect of cluster dynamics, and then I'll leave it to others in this volume to treat the difficult problems in the dynamics of globular clusters.

\section{Inequalities of the Characteristic Times}

I have not called attention to it explicitly, but the ability to treat the dynamics of globular clusters in this simple way is very much dependent on two inequalities:

$$
t_{\mathrm{cr}} \ll t_{\mathrm{rlx}} \ll t_{\mathrm{evol}} .
$$


The quantities involved are the three characteristic times that pertain to the dynamical behavior of the cluster. The first, $t_{\mathrm{cr}}$, is the crossing time, which is the time that a star takes to go from one side of the cluster to the other. (This is clearly an order of magnitude rather than a rigorously defined quantity.)

Next comes the relaxation time $t_{\mathrm{rlx}}$. As I've said earlier, the reference time, in the Fokker-Planck equation, is physically more natural; but the name "relaxation time" is so firmly entrenched in astronomical terminology that I will use that name here. In these inequalities, of course, the small factor by which the two differ doesn't matter.

The third characteristic time, the "evolution time", is the time in which the cluster will change greatly as a result of dynamical evolution. It is clear that this too is a loose definition. Since the principal effect of dynamical evolution is a loss of stars, it might be better to refer to something like a decimation time, but I have never seen that name used.

In a typical globular cluster the inequalities - or maybe because of the double inequality sign I should refer to them as "incomparabilities" - are of the order of a factor of a hundred in each case - although obviously this has to be taken as a loose statement, because the relaxation time, for example, differs a lot from one place in the cluster to another.

The basic steps that I have led you through in reducing cluster dynamics to such simplicity have tacitly assumed these incomparabilities. In talking about how the velocity distribution and the density distribution correspond to each other, I have relied on the crossing time being short compared with the relaxation time. Otherwise we would not be able to talk about an equilibrium at all; by the time a star had traveled across the cluster, the velocity distribution would have changed. And similarly, if the evolution time were comparable with the relaxation time, it wouldn't make sense to talk about a steady-state solution of the Fokker-Planck equation, because as stars were lost, the actors would be changing in the middle of the play.

There is still another circumstance that makes the dynamics of globular clusters simpler than it would otherwise be: the range of masses of stars is not as large as it would be in a younger cluster. Instead of having to deal with a range of stellar masses all the way from the red dwarfs of the lower main sequence up to 50 or 100 solar masses, we go only up to about $0.8 M_{\odot}$, because all the stars more massive than that limit have gone through their main-sequence lives, and nearly all of them are now white dwarfs, whose mass is right in the middle of the range of main-sequence masses.

\section{Unsolved Problems}

In addition to the successes of understanding clusters, I want to conclude by referring to two unsolved problems of cluster dynamics that have bothered me for years.

\subsection{Open clusters}

The first of these is the problem of the dynamics of open clusters, where the inequality $t_{\mathrm{cr}} \ll t_{\mathrm{rlx}}$ is not satisfied. It is easy to show that the ratio of the two times depends mainly on the number of stars in the cluster. At 100 stars, which corresponds to a medium-poor open cluster, the cluster relaxes in just about the time it takes a star to cross it. There is no way then to talk about a dynamical equilibrium.

I have felt for a long time that the way to approach modeling open clusters is to apply Prigogine's principle of a stationary state of higher order (Prigogine 1967), in which the most probable state is the one in which entropy increases at a minimum rate, compared 
with all neighboring states - but I am not aware of anyone ever taking up this problem. (For a discussion of the dynamics of open clusters, see King 1981.)

\subsection{A better treatment of relaxation in a cluster}

At the time I was investigating basic cluster dynamics, I published a paper (King 1960) that took the relaxation process a step closer to reality, by recognizing that the stars were encountering each other, rather than a separate reservoir of stars with Gaussiandistributed velocities. The result made only trivial changes in the resulting velocity distribution, but it made an important change in the physical circumstances. Because the relaxation was produced by encounters between the stars under consideration, the energy of escaping stars had to be removed from other stars in the same distribution. Although the escaping stars carried away zero energy, they left the negative binding energy of the cluster to be shared among fewer stars, with a resulting contraction of the cluster. The steady-state solution described in $\S 3.1$ was then replaced by a self-similar solution, in which the changes in velocity corresponding to the contraction resulted in an escape rate about $50 \%$ higher than in the steady-state solution.

The paper has had absolutely no effect on astronomy. NASA ADS lists 6 citations, all of which merely note the existence of the paper, without any mention of its content. I have always wondered if this reasoning was worth pursuing - or even worthy of being verified by $\mathrm{N}$-body simulations.

\section{Conclusion}

When I was invited to present a paper at this meeting, I thought about what kind of contribution might be most useful, and I chose this review of the basic dynamical nature of globular clusters. There is nothing here that has not been seen before, and for that I apologize to the reader. But as abstruse details accumulate, the simple truths are often lost sight of, and I thought that presenting a reminder of them might be useful.

\section{Acknowledgements}

I would like to acknowledge support from many grants from STScI, especially GO10146, and also a travel grant from the IAU.

\section{References}

Ambartsumian, V. A. 1938, Uchenniye Zapiskiy, Leningrad State Univ., 22, 19 (in Russian) Ambartsumian, V. A. 1985, in: J. Goodman \& P. Hut (eds.), Dynamics of Star Clusters, IAU Symp. No. 113 (Reidel: Dordrecht), p. 521 (English translation of Ambartsumian 1938)

King, I. R. 1960, $A J, 65,122$

King, I. R. 1965, $A J, 70,376$

King, I. R. 1966, $A J, 71,64$

King, I. R. 1981, in: J. Hesser (ed.), Star Clusters, IAU Symp. No. 85 (Reidel: Dordrecht), p. 139

King, I. R. \& Anderson, J. 2001, in: S. Deiters, B. Fuchs, A. Just, R. Spurzem, \& R. Wielen (eds.) Dynamics of Star Clusters and the Milky Way, ASPCS Vol. 228, p. 19

King, I. R. \& Anderson, J. 2002, in: F. van Leeuwen, J. D. Hughes, \& G. Piotto (eds.) $\omega$ Centauri, A Unique Window into Astrophysics, ASPCS Vol. 265, p. 21

Prigogine, I. 1967, Introduction to the Thermodynamics of Irreversible Processes, 3rd edition (New York: Wiley)

Spitzer, L. \& Härm, R. 1958, ApJ, 127, 544 